\title{
WINDS IN THE ATMOSPHERES OF CENTRAL STARS OF PLANETARY NEBULAE
}

\author{
R. P. KUDRITZKI, R. H. MENDEZ AND J. PULS \\ Munich University Observatory
}

AND

J. K. MCCARTHY

Department of Astronomy, California Institute of Technology

\section{Introduction}

For this review we have chosen to concentrate on recent progress concerning the model atmosphere analysis of stellar winds in central stars of planetary nebulae (CSPNs). Since there is another review in these proceedings (by W.R. Hamann) devoted specifically to Wolf-Rayet type CSPNs, we will not consider them here. The reader interested in recent work on windless model atmospheres applied to CSPNs is referred to reviews by Napiwotzki (1995) and Werner et al. (1996). There is also an interesting paper by Werner (1996) on the Balmer line problem.

Although we are ignoring the Wolf-Rayet CSPNs, there is no lack of objects to consider: stellar winds not so spectacular as the Wolf-Rayet kind, but dense enough to be detectable through $P$ Cygni-type profiles of UV resonance lines or through optical and infrared emission lines, are quite common among the best observed CSPNs. If the winds are radiatively driven, they should be conspicuous for all CSPNs sufficiently close to the Eddington limit in the $\log \mathrm{g}-\log T_{\text {eff }}$ diagram. One essential aspect of current work is to verify if the theory can reproduce quantitatively the observed existence, characteristics and behavior of winds in CSPNs, with the same degree of success achieved in the case of massive O, B and A stars (Puls et al. 1996). In this review we try to describe the present situation, which is a mixture of encouraging partial success and some persistent enigmas. 


\section{WINDS IN THE ATMOSPHERES}

\section{Effects of winds on atmospheres}

In principle, all CSPNs evolving towards higher $T_{\text {eff }}$ and more massive than about 0.6 solar masses are expected to have detectable winds at least in the UV and frequently also in the optical spectrum. The radiatively driven winds, if at all detectable in the earlier post-AGB evolution, should become undetectable when the luminosity drops and the star enters a white dwarf cooling track. For such low-luminosity, high-surface-gravity objects, the NLTE hydrostatic models are presumably adequate. On the other hand, as long as the wind is observable, a hydrodynamic, spherically extended model atmosphere, with a certain run of outflow velocity as function of radius, is needed for an adequate description. The presence of a substantial outflow modifies several basic characteristics of the atmosphere as compared to a hydrostatic model (see e.g. Gabler et al. 1989). The density and temperature stratifications are changed. The atmosphere becomes spherically extended, in particular at extreme wavelegths, $\lambda<228 \AA$ and $\lambda>50000$ $\AA$. Infrared and far-UV $(\lambda<228 \AA)$ excesses appear in the spectral energy distribution: the former being very difficult to measure, because of the nebular dust infrared emission, and the latter being important due to the effect of the increased He II ionizing flux on the surrounding nebula. The atomic level populations are changed, and many of the key diagnostic stellar absorption lines are affected and eventually go into emission. All these effects are a function of the mass loss rate. A consistent description of the stellar spectrum requires a simultaneous determination of $T_{\text {eff }}, \log$ $\mathrm{g}$, abundances, stellar radius, terminal wind velocity and mass loss rate; a more difficult situation than we had in the good old days of plane-parallel, hydrostatic NLTE models. The terminal wind velocities can be measured on high-resolution UV spectra obtained with IUE or the Hubble Space Telescope. In next section we consider diagnostics for the mass loss rate.

\section{Mass loss rates of O-type and Of-type CSPNs}

The mass loss rates of CSPNs are more difficult to measure than for massive hot stars. Radio continuum free-free emission from CSPN winds has not been detected up to now, and mass loss rate estimates for CSPNs have been normally based on the modeling of the P Cygni-type profiles of UV resonance lines of highly ionized elements. This method is not very reliable, mostly due to uncertainties in the wind ionization structure. Other non-negligible sources of uncertainty are the distances to PNs and the photospheric abundances of elements heavier than He in CSPNs.

We are currently using an alternative method, which has been successfully applied to the measurement of mass loss rates in massive hot stars. It is based on modeling the $\mathrm{H} \alpha$ stellar profile (Puls et al. 1996 and references 

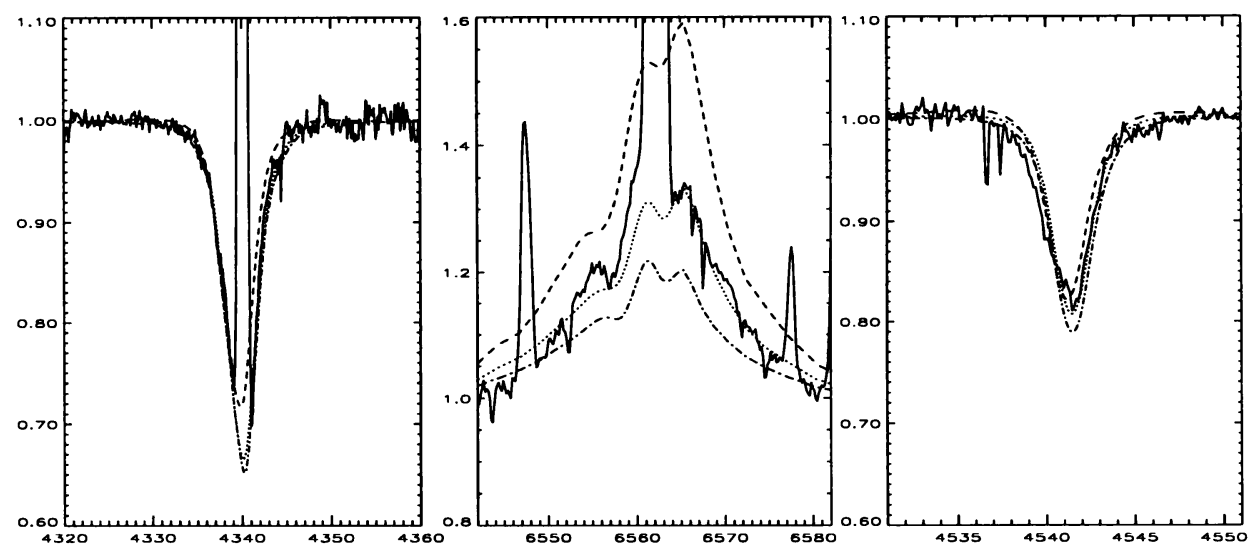

Figure 1. The stellar profiles of $\mathrm{H} \gamma, \mathrm{H} \alpha$ and $\mathrm{He}$ II $\lambda 4541$, calculated with the new unified model atmosphere code developed by Santolaya-Rey, Puls and Herrero (1996) for the following parameters: $T_{\text {eff }}=50000 \mathrm{~K}, \log \mathrm{g}=3.8,(\mathrm{He} / \mathrm{H})=0.15$ by number, $\left(R_{*} / R_{\odot}\right)=2.0, \beta=1.1, v_{\infty}=1200 \mathrm{~km} / \mathrm{s}$, and three mass loss rates: $\dot{\mathrm{M}}=1.9 \times 10^{-7}$ (dash-dotted), $2.5 \times 10^{-7}$ (dotted), and $3.6 \times 10^{-7}$ (dashed) solar masses per year. The observed spectrum corresponds to the central star of NGC 6826. The strength of the stellar $\mathrm{H} \alpha$ emission is very sensitive to $\dot{\mathrm{M}}$.

therein; see also Santolaya-Rey et al. 1996). The strength of the stellar $\mathrm{H} \alpha$, when it is in emission, is a sensitive indicator of the mass loss rate, as shown in Figure 1. In addition, the shape of the profile gives information about the exponent $\beta$ in the expression of the outflow velocity as function of radius, $v(r)=v_{\infty}(1-(b / r))^{\beta}$, where $b$ is a constant selected to yield a prespecified velocity of $0.1 V_{\text {sound }}$ at the transition from the hydrostatic to the hydrodynamic regime.

The fitting procedure is organized as follows: we first make a preliminary estimate of $T_{\text {eff }}, \log \mathrm{g}$ and He abundance using fits to the optical $\mathrm{H}$ and He stellar absorption lines with theoretical profiles computed from planeparallel NLTE models; then we use the preliminary atmospheric parameters, plus an estimate of the stellar mass from evolutionary tracks plotted in the $\log \mathrm{g}-\log T_{\text {eff }}$ diagram, plus the stellar radius derived from the mass and $\log \mathrm{g}$, plus $v_{\infty}$ from the $\mathrm{P}$ Cygni-type profiles of the strongest stellar $\mathrm{UV}$ resonance lines, to calculate fits to the stellar $\mathrm{H} \alpha$ profile for different values of the exponent $\beta$ and the mass loss rate. Having estimated the mass loss rate, we go back and calculate new hydrodynamic "unified" models (always assuming a $\beta$ outflow velocity law, because we have not achieved full physical consistency yet), in order to obtain improved theoretical profiles for the other diagnostic lines, which now incorporate the distorting effects of the wind. The procedure is iterated until we get adequate fits to all stellar absorption and emission lines (with the single exception of the He II 


\section{WINDS IN THE ATMOSPHERES}

$\lambda 4686$ emission, which cannot be modeled very well because it depends in a very subtle way on the treatment of metal line blocking around $304 \AA$, which is the wavelength of the resonance line. The wind models described in this section do not consider metal opacities at all, because they include only $\mathrm{H}$ and $\mathrm{He}$ opacity).

Using the new wind models of Santolaya-Rey et al. (1996) we have tried to estimate how large are the wind effects on the plane-parallel line diagnostics of CSPNs. The result is somewhat unexpected: for weak and average winds the effects are rather small, and only for very strong winds are the plane-parallel masses too large by about 0.1 solar masses. We will come back to this result in Section 4 .

The spectroscopy of the stellar $\mathrm{H} \alpha$ in PNs is not easy, due to the presence of the very strong nebular $\mathrm{H} \alpha$ emission. Fortunately in many cases the stellar emission is broader than the nebular one and can be easily isolated; but sometimes it is very difficult to say if the stellar $\mathrm{H} \alpha$ is in absorption or in emission. Normally in such cases (and also when the stellar $\mathrm{H} \alpha$ is clearly in absorption) we can provide only an upper limit to the mass loss rate.

Table 1 gives some of our results for CSPNs using the iterative procedure described above. Temperatures are given in thousands of $\mathrm{K}, \mathrm{He}$ abundances as number ratios $\mathrm{He} /(\mathrm{H}+\mathrm{He})$, and velocities in $\mathrm{km} / \mathrm{s}$. The observational material has been collected using a variety of telescopes and spectrographs: ESO 3.6m + CASPEC, ESO NTT + EMMI, Isaac Newton 2.5m (La Palma) + IDS, Palomar echelle, McDonald 2.1m + Sandiford echelle. The UV spectrograms were taken with IUE and the Hubble Space Telescope + FOS. The analysis of the UV data is from Haser (1995). Figure 2 shows fits to absorption and emission lines in the spectrum of the central star of IC 4593. A paper, with more information than we can provide here, is in preparation. We estimate that the mass loss rates from $\mathrm{H} \alpha$ have an internal accuracy of \pm 0.1 dex.

Having obtained more reliable mass loss rates, we can investigate to what extent is the radiatively driven wind theory successfully predicting the observed winds.

\section{The relation between stellar wind momentum loss rate and luminosity}

The radiatively driven wind theory predicts, for solar abundances, a simple relation between the quantity $\dot{M} v_{\infty}$, which has dimensions of a momentum loss rate, and the stellar luminosity:

$$
\dot{M} v_{\infty} \sim R_{*}^{-0.5} L_{*}^{(1 / \alpha)}
$$




\section{R.P. Kudritzki}

TABLE 1. Some mass loss rates of CSPNs

\begin{tabular}{lrrrrrrr}
\hline object & $T_{\text {eff }}$ & $\log \mathrm{g}$ & $\dot{\mathrm{M}}\left(M_{\odot} / \mathrm{yr}\right)$ & $\beta$ & $\mathrm{He} \mathrm{ab}$. & $v_{\infty}$ & $R_{*} / R_{\odot}$ \\
\hline He 2-131 & 30 & 2.9 & $9 \times 10^{-7}$ & 1.5 & 0.30 & 500 & 5.5 \\
Tc 1 & 33 & 3.0 & $\leq 1 \times 10^{-7}$ & 1.0 & 0.15 & 900 & 5.1 \\
He 2-108 & 35 & 3.3 & $2.4 \times 10^{-7}$ & 1.5 & 0.20 & 700 & 3.2 \\
IC 418 & 37 & 3.3 & $2.6 \times 10^{-7}$ & 1.5 & 0.20 & 700 & 3.5 \\
IC 4593 & 40 & 3.6 & $1 \times 10^{-7}$ & 1.5 & 0.12 & 900 & 2.2 \\
NGC 2392 & 45 & 3.6 & $\leq 3 \times 10^{-8}$ & 1.5 & 0.25 & 400 & 2.5 \\
NGC 6826 & 50 & 3.8 & $2.6 \times 10^{-7}$ & 1.1 & 0.13 & 1200 & 2.0 \\
IC 4637 & 55 & 4.1 & $\leq 2 \times 10^{-8}$ & $0.7:$ & 0.11 & 1500 & 1.3 \\
NGC 3242 & 75 & 4.7 & $\leq 2 \times 10^{-8}$ & 1.0 & 0.12 & 2300 & 0.6 \\
\hline
\end{tabular}
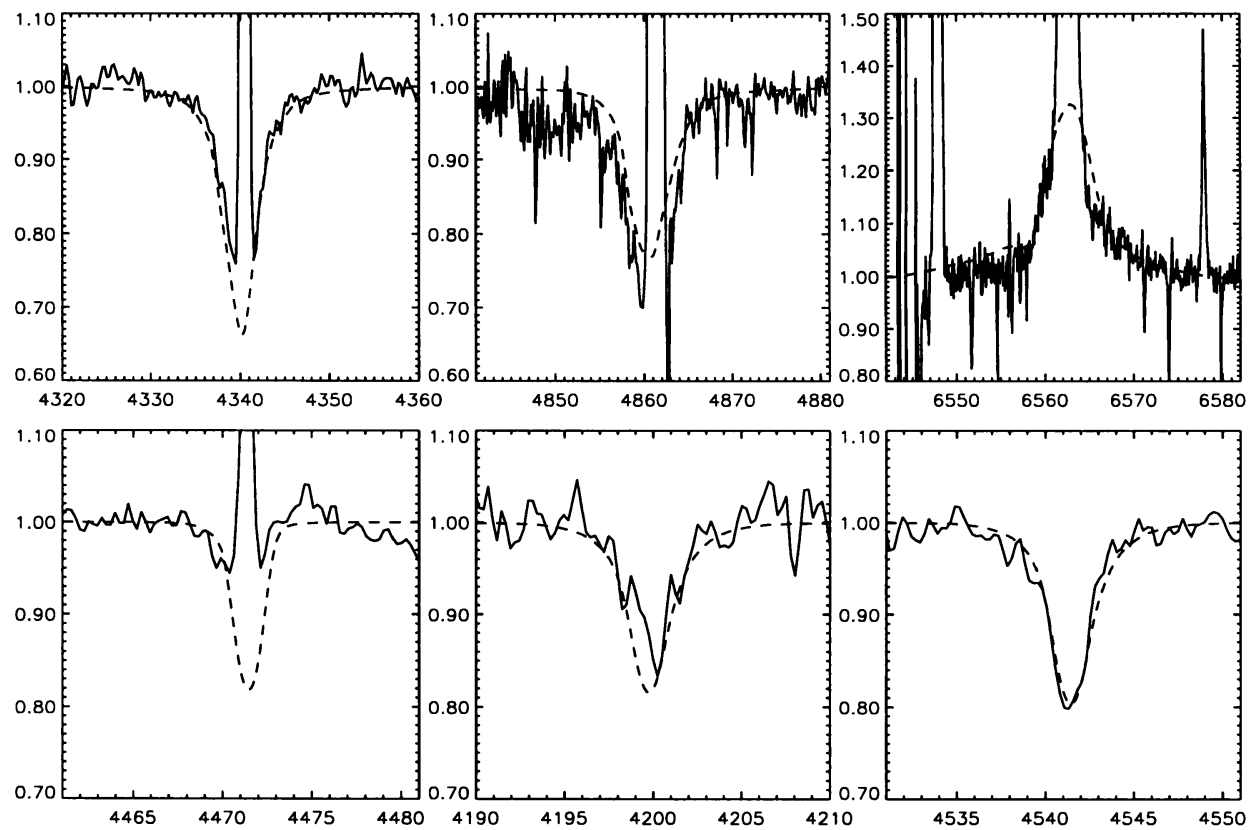

Figure 2. Fits to several absorption and emission features in the spectrum of the central star of IC 4593, using the parameters listed in Table 1. The following lines are fitted: $\mathrm{H} \gamma$, $\mathrm{H} \beta, \mathrm{H} \alpha$, He I 4471, He II 4200 and 4541.

where $\alpha$, the power law exponent of the line strength distribution function, is $\simeq 2 / 3$ (see e.g. Kudritzki et al. 1995 or Puls et al. 1996). It is practical to plot the $\log$ of $\dot{M} v_{\infty} R_{*}^{0.5}$ as a function of $\log L_{*}$. In this kind of plot the theory predicts, in first approximation, a linear relation for solar metallici- 


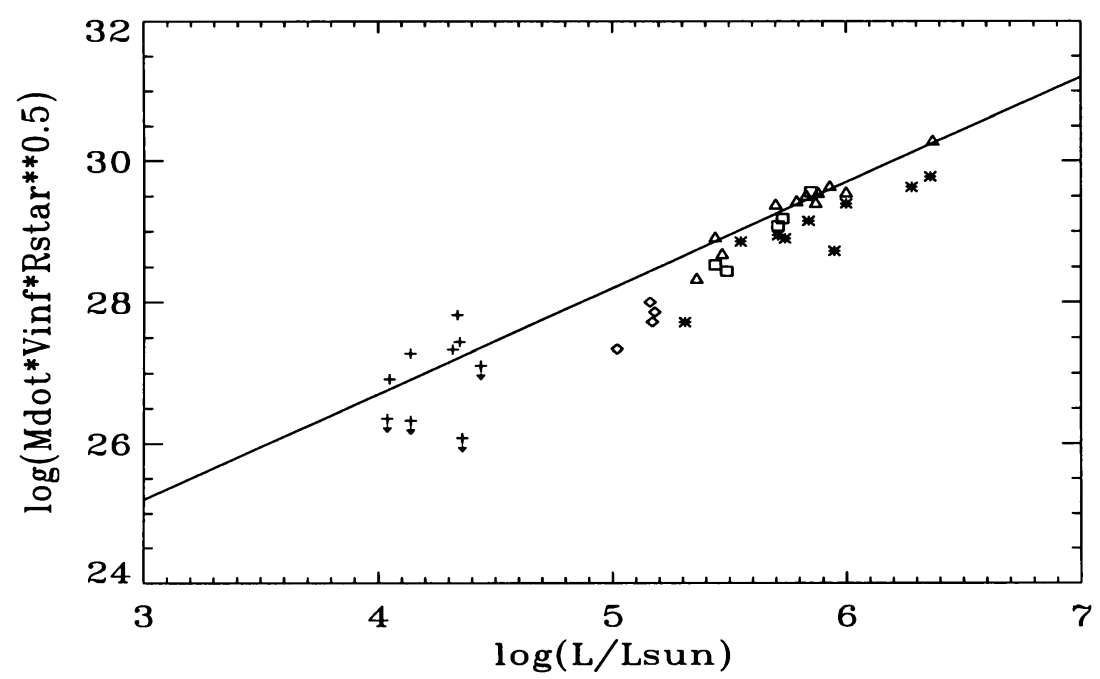

Figure 3. The log of the quantity $\dot{M} v_{\infty} R_{*}^{0.5}$ as function of the log of stellar luminosity, for a variety of hot stars. The numbers along the vertical axis are obtained by expressing $\dot{M} v_{\infty}$ in cgs units and $R_{*}$ in solar radii. Open triangles, squares and diamonds indicate, respectively, massive $\mathrm{O}, \mathrm{B}$ and $\mathrm{A}$ supergiants. Asterisks indicate $\mathrm{O}$ stars of lower luminosity classes, from giants to main sequence objects. Plus signs are for the CSPNs listed in Table 1; the arrows indicate the 4 upper limits. The straight line is the simple relation following the $3 / 2$ power of the stellar luminosity.

ties, which is indeed followed by all kinds of massive hot stars, as shown in Figure 3.

Now we can add several CSPNs in Figure 3: The information about $\dot{M}$, $v_{\infty}$ and $R_{*}$ comes from Table 1 , and the luminosities are derived directly from $T_{\text {eff }}$ and stellar radius. Notice that we do not need PN distances here, although of course spectroscopic distances are implicit in our results.

Several aspects in Figure 3 deserve discussion. First of all, the CSPNs fitted with the procedure described in Section 3 fall very well along the straight line. Therefore, a first look indicates a qualitatively successful prediction by the theory. However, a closer look reveals a few problems. For example, there would seem to be two different groups of CSPNs at low surface gravities, the strong-winded and the faint-winded ones. The prototype of the faint-winded is of course the well-known NGC 2392, but now we find that it is not unique: Tc 1 and IC 4637 are additional examples. Remember that here we are not discussing a third group, the very strong winds of the Wolf-Rayet CSPNs. What is the reason for this variety? One possibility in some cases could be abundance differences (for example, it is quite evident that massive hot stars in the SMC, with a lower average metallicity, have 


\section{R.P. Kudritzki}

systematically weaker winds than in the LMC or in our Galaxy; see Fig. 24 in Puls et al. 1996). We do not know enough about the metallicities of CSPNs to verify if this is a plausible idea, but this problem is certainly one of the strongest motivations to attempt metallicity determinations from the UV spectra of these stars, where $\mathrm{Ni}$ and $\mathrm{Fe}$ lines are abundant; such metallicity determinations are becoming possible at the present time.

Another possibility would be to attribute the different wind strengths to slightly different values of the force multipliers $\mathrm{k}, \alpha, \delta$, in the winds of stars with different spectral types and luminosity classes. In support of this idea we note the slight separation between massive hot stars of different spectral types and luminosity classes in Figure 3; the strong-winded CSPNs would seem to fit better along the relation followed by the $\mathrm{O}$ supergiants, and perhaps the faint-winded CSPNs behave like the asterisks in Figure 3 . These considerations will remain speculative until a more refined wind theory is available.

There is, finally, a more disturbing problem, namely the very high masses and luminosities derived for some CSPNs using the new hydrodynamic models. In some cases the new stellar luminosities and masses are comparable to the hydrostatic values obtained earlier by Méndez et al. (1988). This is surprising. We had expected the new models to confirm the reduced masses estimated by Méndez et al. (1992), but apparently this is not always the case. As they stand, the highest luminosities we have obtained are in contradiction with theoretical post-AGB evolutionary speeds (according to which we should expect to find all such massive objects at much lower luminosities, along white dwarf cooling tracks).

On the other hand, the current models need such high luminosities to produce the successful location of CSPNs along the straight line in Figure 3 . If we reduce the luminosities, although $\dot{M}$ and $R_{*}$ are also reduced, anyway the CSPNs are displaced towards the region above the straight line, and some CSPN winds become much stronger than expected from the radiatively driven wind theory as we can implement it now. This would become another manifestation of the same problem we know very well from the Wolf-Rayet stars: they also lie above the straight line in Figure 3. Since our models are not yet fully physically consistent, we cannot rule out the possibility that, due to some missing physical ingredient that strengthens the winds, we are led to overestimate the CSPN radii and luminosities in our effort to fit wind-sensitive features. In conclusion, although there has been some significant progress, we do not fully understand the CSPN winds yet. In next Section we consider if NLTE metal-line blanketing can offer a solution. 


\section{WINDS IN THE ATMOSPHERES}

\section{Winds and NLTE metal-line blanketing}

At the time of the previous (Innsbruck) Symposium, work on NLTE metalline blanketing was just starting. Nowadays it is becoming a real thing. Concerning hydrostatic, plane-parallel NLTE line blanketed models including millions of metal lines, most of the activity takes place in Germany (Kiel, Bamberg, Potsdam: Werner, Dreizler, Haas, Heber et al.) and in the U.S.A. (Goddard: Hubeny, Lanz, Heap, deKoter et al.). This kind of models is applicable to hot subdwarfs, low-mass CSPNs, very hot white dwarfs and late $\mathrm{O}$ main sequence stars.

Concerning the even more ambitious hydrodynamic (winds, spherical extension) NLTE metal-line blanketed models with millions of lines, there are active groups in Geneva and Zürich (Schaerer, Schmutz), Pittsburgh (Hillier, Miller) and Munich (Kudritzki, Pauldrach, Puls, Sellmaier). This kind of models should be applicable to all kinds of hot stars, but in particular it is mostly needed for massive $\mathrm{O}, \mathrm{B}, \mathrm{A}$ supergiants, high-mass CSPNs, Wolf-Rayet stars of all kinds, novae, supernovae, and post-AGB proto-CSPNs (more about the last ones in next Section).

Here we can only make a brief summary of some recent results. The energy distributions are (as expected) strongly affected by NLTE metal-line blanketing, and this has interesting effects on some nebular diagnostics. For example, Sellmaier et al. (1996) have shown that the energy distributions of hydrodynamic NLTE metal-line blanketed models can solve the [Ne III] problem in H II regions (which we cannot describe here; see the reference). We expect that in the near future the new energy distributions will become the standard input for nebular photoionization models.

We have not yet made a detailed line profile analysis of any CSPN with the new hydrodynamic NLTE metal-line blanketed models. However, we can report that the UV spectra of massive Of stars like $\zeta$ Puppis are very well represented, and we have compared the profiles of several key optical diagnostic lines (Balmer, He I, He II) against profiles from the unblanketed wind models we mentioned in Section 3. At temperatures between 30000 and $50000 \mathrm{~K}$, the comparison shows that Balmer lines are unaffected and $\mathrm{He}$ II lines like $\lambda 4541$ are weakly affected. The differences are more noticeable for He I lines (blanketing makes them weaker). These results imply that line profile fits with the new NLTE line-blanketed models will produce slight shifts in the positions of CSPNs in the $\log g-\log T_{\text {eff }}$ diagram, but with negligible effects on the derived stellar masses and luminosities. This is disappointing, given the problem with these quantities described in Section 4 above. 


\section{R.P. Kudritzki}

\section{Atmospheres of post-AGB proto-CSPNs}

In recent years, several dozens of stars of spectral type $G$ to $B$ and very low surface gravity (unfortunately misnamed supergiants) have been identified as post-AGB stars (see e.g. Parthasarathy 1993). Although most of these stars are known to have winds, the spectral analyses made up to now have used plane-parallel, hydrostatic model atmospheres. Therefore it is important to remark that the effects of atmospheric extension become very strong at low $T_{\text {eff }}$, so that in these cases hydrodynamic, spherically extended models are necessary for the accurate determination of basic stellar atmospheric parameters. We have made some estimates along the postAGB evolutionary tracks, showing that even for masses of $0.6 M_{\odot}$ there are, at temperatures of $10000 \mathrm{~K}$, significant sphericity effects.

$\mathrm{H} \alpha$ observations in some of these objects can provide, in the same way as in hotter cases, quite accurate values of $\dot{M}$. One example is HD 172324, a post-AGB star classified as A0 Ia. Using a spectrum taken with the Palomar echelle we could estimate from $\mathrm{H} \alpha$ a mass loss rate of $10^{-8} M_{\odot} / \mathrm{yr}$.

\section{CSPNs with strong winds in the Galactic bulge}

Studies of planetary nebulae in the Galactic bulge are important for a thorough understanding of stellar and Galactic evolution. Up to now the properties of their central stars have been derived using Zanstra temperatures and making somewhat controversial assumptions about the uniformity of nebular masses and expansion velocities, or assuming that most PNs are completely optically thick in the $\mathrm{H}$ Lyman continuum, i.e. that there is no leakage of ionizing radiation. It would be interesting to investigate if the bulge central stars differ in any systematic way from the CSPNs in the solar neighborhood, but in the present conditions it is not easy to make a convincing comparison. We believe that the only way to make progress is to undertake direct spectroscopic studies of the bulge CSPNs. Therefore we have initiated a spectroscopic survey of the brightest bulge CSPNs, taking high-resolution optical spectrograms with the Keck telescope + HIRES spectrograph. We have selected a sample of low-excitation PNs in the direction of the galactic center, because in such cases (1) we know the stars are evolving at high luminosities towards higher temperatures, and (2) it is easier to make an accurate determination of $T_{\text {eff }}$ from He I and He II features.

The spectra are of good quality and the analysis is in progress. One of the first things we have noticed is that several CSPNs in the bulge, with normal $\mathrm{He}$ abundances (i.e. not $\mathrm{H}$-deficient), have very strong winds: some examples are $\mathrm{M}$ 1-37, $\mathrm{H}$ 1-35 and $\mathrm{H}$ 1-65. Figure 4 shows some bulge CSPNs arranged in order of increasing wind strength. These observations are an 


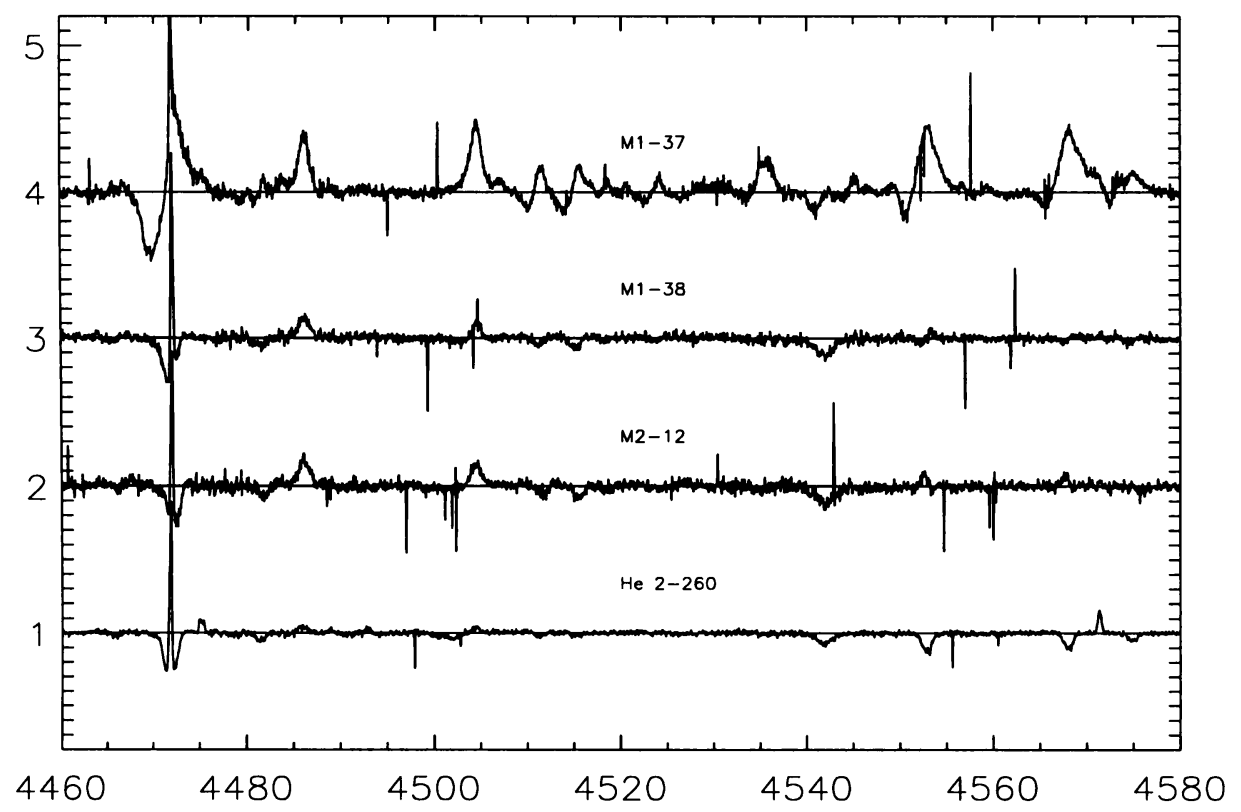

Figure 4. A short section of our Keck HIRES spectrograms of 4 bulge CSPNs. The most important features are He I 4471, He II 4541, Si III 4552, 4567, 4574 and the unidentified emissions at 4485 and 4503 . The strong $P$ Cygni-type profiles in M 1-37 are remarkable.

ideal ground for the application and further testing of the new hydrodynamic models, and we expect to learn quite a lot from a direct spectroscopic comparison between solar neighborhood and bulge CSPNs.

\section{References}

Gabler R., Gabler A., Kudritzki R.P., Puls J., Pauldrach A. 1989, A\&A 226, 162 Haser S.M. 1995, Ph.D. Thesis, Munich.

Kudritzki R.P., Lennon D.J., Puls J. 1995, in Procs. of ESO Workshop Science with the $V L T$, eds. J.R. Walsh and I.J. Danziger, Springer, p. 246

Méndez R.H., Kudritzki R.P., Herrero A. 1992, A\&A 260, 329

Méndez R.H., Kudritzki R.P., Herrero A., Husfeld D., Groth H.G. 1988, A\&A 190, 113

Napiwotzki R. 1995, in White Dwarfs: Springer Lecture Notes in Physics 443, 176

Parthasarathy M. 1993, in Luminous High-latitude Stars: ASP Conference Series 45, 173

Puls J., Kudritzki R.P., Herrero A., Pauldrach A. et al. 1996, A\&A 305, 171

Santolaya-Rey A.E., Puls J., Herrero A. 1996, A\&A (submitted)

Sellmaier F.H., Yamamoto T., Pauldrach A., Rubin R.H. 1996, A\&A 305, L37

Werner K. 1996, ApJ 457, L39

Werner K., Dreizler S., Heber U., Rauch T. 1996, in H-Deficient Stars: ASP Conference Series 96,267 